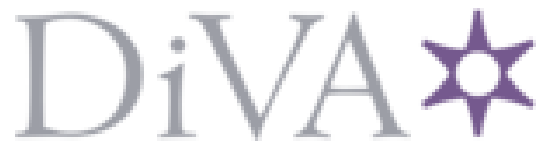

http://www.diva-portal.org

Preprint

This is the submitted version of a paper published in Current opinion in structural biology.

Citation for the original published paper (version of record):

Ivarsson, Y., Jemth, P. [Year unknown!]

Affinity and specificity of motif-based protein-protein interactions

Current opinion in structural biology

Access to the published version may require subscription.

N.B. When citing this work, cite the original published paper.

Permanent link to this version:

http://urn.kb.se/resolve?urn=urn:nbn:se:uu:diva-362357 
Affinity and specificity of motif-based protein-protein interactions

Ylva Ivarsson $^{1}$ and Per Jemth ${ }^{2}$

${ }^{1}$ Department of Chemistry-BMC, Uppsala University, Box 576, SE-751 23 Uppsala, Sweden.

${ }^{2}$ Department of Medical Biochemistry and Microbiology, Uppsala University, BMC Box 582, SE-75123 Uppsala, Sweden. 
It is becoming increasingly clear that eukaryotic cell physiology is largely controlled by protein-protein interactions involving disordered protein regions, which usually interact with globular domains in a coupled binding and folding reaction. Several protein recognition domains are part of large families where members can interact with similar peptide ligands. Because of this, much research has been devoted to understanding how specificity can be achieved. A combination of interface complementarity, interactions outside of the core binding site, avidity from multidomain architecture and spatial and temporal regulation of expression resolve the conundrum. Here, we review recent advances in molecular aspects of affinity and specificity in such protein-protein interactions.

How can proteins distinguish their biologically relevant binding partner from other proteins in the cell? The answer is that the affinity of the protein-protein interaction under a given set of conditions is high enough compared to all other competing interactions. However, teasing out the details of the higher affinity for a certain protein-protein interaction is far from simple since multiple factors govern how much will be bound. We can measure the affinity using isothermal calorimetry and even dissect it into the change in strength of all individual bonds (enthalpy) and the change in entropy of the whole system, including release of water and ions upon binding, but this requires extensive experiments to give insight. Because of the complexity of even the simplest protein-protein interaction, from an experimental point of view it is often easier and more informative to assess differences in affinities, i.e., specificity, for proteins with several binding partners. In other words, why is a 
certain interaction favored over another one? Such competition between different binding partners is also what happens in the crowded milieu of the cell. In vivo, spatial and temporal regulation contribute to resolving the apparent problem of overlapping specificity [1]. In this review we will focus on molecular aspects of affinity and specificity of motif-based interactions, including short linear motifs and coupled binding and folding of intrinsically disordered regions, and how they can prevail in the context of multiple competing interactions (Figure 1). The topic is of relevance for a fundamental understanding of the molecular events of endogenous processes but also from the perspective of targeting motif-based interactions for therapeutic purposes, a topic that has been receiving increasing attention in recent years [2], with the recently developed ABT-199 inibitor of BCL-2 as a prime example [3]. A classical function of interactions mediated by disordered binding motifs is to serve as binding sites for the assembly of protein complexes, often in the context of scaffolding proteins in signaling pathways $[4,5]$. Other functions involve guiding proteins to subcellular compartments (e.g. EB family [6]), docking of enzymes to their substrates, (e.g. MAPKSs [7,8], PP2A [9,10], Calcineurin [11], recruitment of transactivation domains to the transcriptional machinery [12-14], or the targeting of proteins for ubiquitin dependent degradation by so-called degrons $[15,16]$. Clearly, there are different requirements on the affinity and specificity of these various motifbased interactions.

\section{Affinity and specificity of motif-based interactions}

Many protein-protein interactions involve binding and folding of intrinsically disordered, i.e., structurally heterogeneous regions of proteins to a folded protein domain, or less commonly to another disordered region. The shorter disordered 
binding regions (3-12 residues) are called short linear motifs (SLiMs), or eykaryotic linear motifs (ELMs), and are extremely common in proteomes of eukaryotic organisms. To give an idea of the numbers, more than 100 domain families have been reported to bind to SLiMs [17], and the number of SLiMs involved in protein-protein interactions in the human proteome has been estimated to be as many as 100,000 [18]. A binding motif is typically visualized as a linear string, with the main determinants of binding indicated using the one letter amino acid code, and positions of less importance indicated with " $x$ ". Upon binding, disordered regions adopt more defined conformations, such as an $\alpha$-helix, a $\beta$-sheet or a less well defined secondary structure, but sometimes remain structurally heterogeneous [19]. Thus, while graphically attractive the simplistic linear-string visualization of SLiMs in disordered regions only highlights side chains but not the tertiary structure adopted upon binding, which obviously influence affinity and specificity in the interactions. Motif based interactions often show apparently low specificity, i.e., several different SLiMs can bind to the same domain with similar affinities, and several domains can bind to the same SLiM. Clearly, it is challenging to achieve specificity in large domain families such as $\mathrm{SH} 2, \mathrm{SH} 3$ and PDZ, all with more than 100 members, which share structural features in their binding interface and inevitably ligand specificity. However, domain family wide studies have shown that there are gradients of specificities within the families that might contribute to the functional specificity, e.g., for PDZ [20,21], SH2 [22] and SH3 [23]. Furthermore, several of the domains of a given family may bind to atypical or "non-canonical" motifs [23]. Such interactions could be the physiological relevant ones or represent moonlighting activities, which are likely very common and a potential source for evolution of new protein-protein interactions [24,25]. 
For motif-based interactions, affinity and specificity are accomplished by a combination of core motif binding determinants that provide shape and/or chemical complementarity, together with the context of the motif within the sequence, and the dynamic properties of the system including concentrations of interacting species (Fig 1, Box 1). The residues of the core motif generally interact with a set of conserved residues in the binding site of the domain. As a consequence of the limited binding interfaces (typically $500 \AA^{2}$ ), SLiM interactions typically have $K_{\mathrm{d}}$ values in the range 1-500 $\mu \mathrm{M}$, which is considered "medium-to-low affinity". Such affinities are usually associated with high dissociation rate constants and thus a rapid equilibrium [26,27]. Affinities in this range are often, but not always, associated with relaxed specificities. This is however not a limitation set by the interaction type as the affinities and specificities of the domains and the motifs are easily evolvable [28], but rather a result of the functional demands on the interactions. Longer disordered binding motifs can have higher affinities [29] yet with high dissociation rate constants allowing equilibrium to be established rapidly enough for the cellular function (within seconds) $[30,31]$.

\section{BOX 1: Affinity, specificity and multivalency}

(a) The affinity is usually quantified and expressed as the equilibrium dissociation constant, $K_{\mathrm{d}}$, which has the unit of $\mathrm{M}$ for an interaction involving two proteins. (b) Specificity is qualitatively used to describe that a certain protein recognizes a certain molecule better than other ones. Sometimes "selectivity" is used to indicate that a protein has several ligands, thus reserving "specificity" for the cases where there is only one ligand. However, perfect specificity is rarely encountered in biological systems and we use the two words as synonyms. A straightforward quantitative 
definition of specificity is the ability of a protein $\mathrm{P}$ to distinguish between competing protein ligands $\mathrm{L}_{1}$ and $\mathrm{L}_{2}$, or vice versa, a ligand $\mathrm{L}$ to distinguish between two proteins $\mathrm{P}_{1}$ and $\mathrm{P}_{2}$. This is expressed as the ratio of the $K_{\mathrm{d}}$ values for the two ligands: Specificity $=K_{\mathrm{d}}^{\mathrm{A}} / K_{\mathrm{d}}^{\mathrm{B}}$

The advantage of looking at specificity rather than affinity is that affinity is highly dependent on the conditions: $\mathrm{pH}$, ionic strength, crowding etc. While specificity may also be modulated by these factors, it is likely that several of them affect the two interactions similarly and cancel out. Thus, in vivo a $K_{\mathrm{d}}$ value may differ much from that measured in vitro. But a 10 -fold higher $K_{\mathrm{d}}$ for protein $\mathrm{P}_{1}$ relative to protein $\mathrm{P}_{2}$ in the test tube is more likely to remain 10 -fold higher in vivo than the absolute $K_{\mathrm{d}}$ values. To obtain the relative abundance of the respective complex the concentrations need to be taken into account as shown in (b). Thus, a high affinity, "specific" interaction could be outcompeted by a low affinity interaction if the concentration of the latter is high enough. Protein concentrations vary between different cell types, cell states and compartments. There are emerging techniques to quantify protein concentration with spatial resolution, for example mass spectrometry and multiplexed immunofluorescent staining [32]. (c) The affinity of multivalent interactions can be quantified by defining $K_{\mathrm{d}}$ as the product of free species divided by the sum of all bound species (two bound species in case of a 1:1 binding with mutually exclusive binding sites or three bound species in the case of the depicted bivalent interaction). (d) Allovalency is an extension of multivalency in which one more factor is taken into account: the escape from the common sphere where protein and ligand can re-bind with higher probability than two fully separated molecules [33,34]. PDB codes of structures used: 5VWK (a, b and d), 3QJN (yellow, b) and 3FM7 (c). 
Given the variety of interactions and the conformational plasticity of disordered interaction regions, it is not surprising that there is no unique solution for how to accomplish specificity of motif-based interactions. Granting that the motif is defined by the core residues, the local context of the motif contributes to affinity. For example, for disordered motifs that adopt $\alpha$-helical structures upon binding, it has been found that increasing the helical propensity of the disordered region confers an increase in affinity for the binding partners [35-38]. Motif-flanking regions can also contribute with specificity determining information in terms of permissive amino acids that increase the affinity of the interaction [39-42]. Indeed, a systematic analysis of structures of motif-based interactions revealed that the local sequence context contributes about $20 \%$ of the binding and is crucial for determining specificity [40]. As an example, the regulatory subunit B56 of protein phosphatase 2 recognizes an LxxIxE motif [9], which is reinforced by the presence of acidic residues located Cterminally of the motif, and/or by phosphorylation within and/or adjacent to the motif $[9,10,43]$. This enhancement of affinity is accomplished by electrostatic interactions between these negatively charged moieties and a basic patch on B56. Similarly, the affinity and specificity of the retinoblastoma protein for the $\mathrm{LxCxE}$ motif is enhanced by negatively charged residues, or phosphorylation of residues situated C-terminally of the core binding motif that interact with a positively charged surface surrounding the binding site [44]. Another recent study showed that phosphorylation or phosphomimetic mutations (S/T to E) within or N-terminally of the class I PDZ binding motifs (T/Sx $\Phi$-coo-) can tune the selectivity of the peptide ligand, such that it enhances the affinity of one PDZ domain while reducing the affinity of another PDZ domain [45]. The effect can in part be explained by the presence or absence of basic residues surrounding the peptide binding pocket of the domain. The contextual 
specificity determinants can also function by negative selection, in other words, nonpermissive residues reduce the affinity of the interaction for certain proteins. One example of achieving high specificity by such gatekeeping is the PxxP motif of the yeast protein $\mathrm{Pbs} 2$ that among all yeast $\mathrm{SH} 3$ domains only binds to the $\mathrm{Sho} 1 \mathrm{SH} 3$ domain, but cross reacts with $\mathrm{SH} 3$ domains from other species [46]. The motif has evolved to bind one specific yeast SH3 domain not by optimizing the affinity for the target domain, but by negative selection against all other SH3 domains in yeast.

Importantly, the information content in the flanking regions is not captured in the core binding motif. Thus, although the SLiM is known, it is challenging to accurately predict ligands of motif-binding proteins, or conversely, predict motifbinding proteins for a certain ligand. Any such predictions therefore requires experimental validation [47]. Predictions of motif-based protein-protein interactions can be improved by taking evolutionary information into consideration [48], given that functional motifs are subjected to evolutionary constraints and are more conserved than the surrounding intrinsically disordered regions in which they are typically embedded. When available, predictions can be improved when taking into consideration contextual information on non-permissive residues $[42,49]$. Nevertheless, experimental methods such as various display methods and peptide SPOT arrays remain in our opinion, more powerful than motif-based predictions in terms of finding interactions and exploring the specificity of motif-based interaction [50-58]. Regardless of the approach used to identify potential endogenous motifbased interactions, it is necessary to confirm that the identified binding site is available for binding in the context of the full-length proteins and that the suggested interacting proteins can meet in the cell [47], which brings us to the next level of the contribution of the context to specificity. 


\section{The larger context}

In addition to the core binding motif and the local context, the larger context contributes to affinity and specificity in terms of potential interactions between other parts of the full-length proteins. For example, intrinsically disordered regions of proteins that contain multiple recognition motifs can interact with two or more domains of a multidomain protein, or to different subunits of a multimeric protein $[59,60]$. Such multivalent interactions are common for motif-based interactions [61] and give rise to avidity effects [62] due to the high local concentration of the second binding site after the initial binding, and the rebinding upon dissociation of individual binding sites (Box 1). Viral proteins often contain multiple motifs used to hijack different human protein-protein interactions [63-65]. In such cases the avidity effect could potentially be explored by designing multivalent inhibitors towards the viral protein by combining parts of the different human targets in a "protein drug" $[66,67]$.

Another specificity enhancing effect is allovalency, which is augmented by the presence of multiple weak affinity bindings sites on an intrinsically disordered protein that binds to one unique binding site (Fig. 1, Box 1). The high local concentration of multiple binding sites results in several binding-dissociation events between the same two molecules $[33,34]$ as illustrated using multiple phosphorylation sites binding to a receptor [68]. In this way multi-site phosphorylation was suggested to lead to ultrasensitive switches that depend on the number of binding sites available. However,arcu few examples are reported. In a recent study, it was shown that the Cul E3 ligase substrate adaptor KBTBD8 requires phosphorylation of multiple E3 binding motifs located in the intrinsically disordered regions to specifically interact with its target proteins [69] While clearly multivalent, whether or not allovalent effects are present 
could be difficult to determine since the presence of multiple binding sites in itself will increase the apparent affinity.

Contributing to the larger context are other factors (Box 1), such as protein concentrations and cellular localization, which depend on cell type and cell state, but a detailed analysis of this is beyond the scope of this review.

\section{Evolutionary aspects}

The types of protein-protein interactions discussed here have been instrumental in the evolution of multicellular life. In particular, re-wiring of interaction networks by shuffling of interaction domains and alternative splicing can open up or close signaling pathways $[70,71]$. But evolution can also operate on the level of the single protein-protein interaction. It has been demonstrated that it is possible to alter the peptide binding specificity of a given domain by single amino acid substitutions [72], to generate domains with novel binding specificities $[73,74]$ and to generate "superbinding" peptides with higher affinity than naturally occurring ligands [75] through the substitutions of a few amino acids. These findings highlight the plasticity of motif-based interactions and reinforce that native interactions have not evolved to be of optimal affinity. However, a recurring observation is that specificity is lost when domains are subjected to in vitro evolution experiment for higher affinity. For example, it was shown by high-throughput phage display that many different binding sites can be generated to bind to a given peptide, but that the artificially generated binders recognize their cognate ligands with high affinity but with low specificity [76]. In another study an engineered PDZ domain selected for binding a viral peptide [66] gained in affinity but lost specificity [77]. 
The motif themselves can rapidly evolve and emerge de novo from a few amino acid substitutions, as extensively reviewed recently [55]. For example, the substrates of the protein phosphatase Calcineurin in yeast and mammals differ due to recently evolved PxIxT sites, which has led to network rewiring [78]. Importantly, while evolution of the core binding motif is crucial, the context also needs to be evolutionary optimized to increase affinity and specificity. Sometimes non-specific interactions serve as starting points for de novo motif evolution. In fact, the high concentrations of proteins in the cell leads to extensive non-specific "quinary interactions" [79]. A few of these very low affinity interactions may provide a specific advantage for the organism and any point mutation, which increases the affinity will then be under positive selection. This can be seen for viruses, where there are numerous examples of how relatively few substitutions can turn a disordered protein region into a SLiM mimicking one of the partners in a protein-protein interaction in the host $[24,80]$. Because viruses evolve rapidly such adaptations can occur on very short timescales. However, similar evolutionary adaptations of new or existing protein-protein interactions involving SLiMs or longer disordered regions probably occur in all life, but on longer timescales. For example, the interaction between the two transcriptional coregulators, CREB-binding protein and NCOA, likely started as a low affinity interaction between the NCBD domain in CREB-binding protein and a disordered region in NCOA [25].

\section{Concluding remarks}

It is a daunting task to fully understand the interaction network of our $>100,000$ binding motifs and their partners. Protein concentrations are regulated depending on cell type, spatially within the cell and over time to coordinate life processes. The 
structure and function of protein-protein interactions are addressed by several approaches and their combination in systems biology will produce ever better pictures of the entire interaction network in the coming decades. Here we have discussed recent results at the molecular end of the question, namely how the first level of specificity is achieved in the coupled binding and folding reactions of intrinsically disordered interaction regions. It is clear that the "core binding motif" of a particular family of interactions is not sufficient to provide specificity, but it relies on further interactions close to the binding motif and between neighboring domains and other proteins [81]. In the future, this highly active area of research should quantitatively address specificity in protein-protein interaction networks. This needs to be done at several levels: for purified proteins, in cells and ultimately in vivo.

\section{Acknowledgements}

The authors are grateful to Elias Tjärnhage who made the illustrations. YI and PJ acknowledge support from the Swedish foundation for strategic research (SB16-0039) and the Swedish research council (2016-04965 to YI and 2016-04134 to PJ).

\section{References}

1. Good MC, Zalatan JG, Lim WA: Scaffold proteins: hubs for controlling the flow of cellular information. Science 2011, 332:680-686.

2. Corbi-Verge $\mathrm{C}$, Kim PM: Motif mediated protein-protein interactions as drug targets. Cell Commun Signal 2016, 14:8.

3. Souers AJ, Leverson JD, Boghaert ER, Ackler SL, Catron ND, Chen J, Dayton BD, Ding H, Enschede SH, Fairbrother WJ, et al.: ABT-199, a potent and selective BCL-2 inhibitor, achieves antitumor activity while sparing platelets. Nat Med 2013, 19:202-208. 
4. Bhattacharyya RP, Reményi A, Yeh BJ, Lim WA: Domains, motifs, and scaffolds: the role of modular interactions in the evolution and wiring of cell signaling circuits. Annu Rev Biochem 2006, 75:655-680.

5. Van Roey K, Uyar B, Weatheritt RJ, Dinkel H, Seiler M, Budd A, Gibson TJ, Davey NE: Short linear motifs: ubiquitous and functionally diverse protein interaction modules directing cell regulation. Chem Rev 2014, 114:6733-6778.

6. Akhmanova A, Steinmetz MO: Control of microtubule organization and dynamics: two ends in the limelight. Nat Rev Mol Cell Biol 2015, 16:711-726.

7. Tanoue T, Adachi M, Moriguchi T, Nishida E: A conserved docking motif in MAP kinases common to substrates, activators and regulators. Nat Cell Biol 2000, 2:110-116.

8. Zeke A, Bastys T, Alexa A, Garai Á, Mészáros B, Kirsch K, Dosztányi Z, Kalinina OV, Reményi A: Systematic discovery of linear binding motifs targeting an ancient protein interaction surface on MAP kinases. Mol Syst Biol 2015, 11:837.

9. Hertz EPT, Kruse T, Davey NE, López-Méndez B, Sigurðsson JO, Montoya G, Olsen JV, Nilsson J: A Conserved Motif Provides Binding Specificity to the PP2A-B56 Phosphatase. Mol Cell 2016, 63:686-695.

10. Wu C-G, Chen H, Guo F, Yadav VK, Mcilwain SJ, Rowse M, Choudhary A, Lin $\mathrm{Z}$, Li Y, Gu T, et al.: PP2A-B' holoenzyme substrate recognition, regulation and role in cytokinesis. Cell Discov 2017, 3:17027.

11. Roy J, Cyert MS: Cracking the phosphatase code: docking interactions determine substrate specificity. Sci Signal 2009, 2:re9.

12. Ptashne M, Gann A: Transcriptional activation by recruitment. Nature 1997, 386:569-577.

13. Staller MV, Holehouse AS, Swain-Lenz D, Das RK, Pappu RV, Cohen BA: A High-Throughput Mutational Scan of an Intrinsically Disordered Acidic Transcriptional Activation Domain. Cell Syst 2018, 6:444-455.e6.

14. Ravarani CN, Erkina TY, De Baets G, Dudman DC, Erkine AM, Babu MM: High-throughput discovery of functional disordered regions: investigation of transactivation domains. Mol Syst Biol 2018, 14:e8190.

15. Mészáros B, Kumar M, Gibson TJ, Uyar B, Dosztányi Z: Degrons in cancer. Sci Signal 2017, 10.

*16. Koren I, Timms RT, Kula T, Xu Q, Li MZ, Elledge SJ: The Eukaryotic Proteome Is Shaped by E3 Ubiquitin Ligases Targeting C-Terminal Degrons. Cell 2018, 173:1622-1635.e14.

The discovery of distinct C-terminal degrons recognized by Cullin-Ring E3 ligases using a global stability approach demonstrates the importance of establishing the roles of motif-based interactions for understanding cell function. 
17. Gouw M, Michael S, Sámano-Sánchez H, Kumar M, Zeke A, Lang B, Bely B, Chemes LB, Davey NE, Deng Z, et al.: The eukaryotic linear motif resource 2018 update. Nucleic Acids Res 2018, 46:D428-D434.

18. Tompa P, Davey NE, Gibson TJ, Babu MM: A million peptide motifs for the molecular biologist. Mol Cell 2014, 55:161-169.

19. Fuxreiter M: Fuzziness in Protein Interactions-A Historical Perspective. $J$ Mol Biol 2018, 430:2278-2287.

20. Tonikian R, Zhang Y, Sazinsky SL, Currell B, Yeh J-H, Reva B, Held HA, Appleton BA, Evangelista M, Wu Y, et al.: A specificity map for the PDZ domain family. PLoS Biol 2008, 6:e239.

21. Vincentelli R, Luck K, Poirson J, Polanowska J, Abdat J, Blémont M, Turchetto J, Iv F, Ricquier K, Straub M-L, et al.: Quantifying domain-ligand affinities and specificities by high-throughput holdup assay. Nat Methods 2015, 12:787-793.

22. Tinti M, Kiemer L, Costa S, Miller ML, Sacco F, Olsen JV, Carducci M, Paoluzi S, Langone F, Workman CT, et al.: The $\mathbf{S H 2}$ domain interaction landscape. Cell Rep 2013, 3:1293-1305.

23. Teyra J, Huang H, Jain S, Guan X, Dong A, Liu Y, Tempel W, Min J, Tong Y, Kim PM, et al.: Comprehensive Analysis of the Human SH3 Domain Family Reveals a Wide Variety of Non-canonical Specificities. Structure 2017, 25:1598-1610.e3.

24. Davey NE, Cyert MS, Moses AM: Short linear motifs - ex nihilo evolution of protein regulation. Cell Commun Signal 2015, 13:43.

*25. Hultqvist G, Åberg E, Camilloni C, Sundell GN, Andersson E, Dogan J, Chi $\mathrm{CN}$, Vendruscolo M, Jemth P: Emergence and evolution of an interaction between intrinsically disordered proteins. eLife 2017, 6:e16059.

Ancestral sequence reconstruction in combination with biophysical methods were used to track the coevolution of both partners in a protein-protein interaction.

26. Gianni S, Jemth P: How Fast Is Protein-Ligand Association? Trends Biochem Sci 2017, 42:847-849.

*27. Bagshaw CR: Biomolecular kinetics: a step-by-step guide. CRC Press, Taylor \& Francis Group; 2017.

This book is a must read for everyone who is interested in theory, methodology and interpretation of kinetic experiments and wants to use it to understand protein-protein interactions.

28. Kaneko T, Sidhu SS, Li SSC: Evolving specificity from variability for protein interaction domains. Trends Biochem Sci 2011, 36:183-190. 
29. Wright PE, Dyson HJ: Intrinsically disordered proteins in cellular signalling and regulation. Nat Rev Mol Cell Biol 2015, 16:18-29.

30. Zhou H-X: Intrinsic disorder: signaling via highly specific but short-lived association. Trends Biochem Sci 2012, 37:43-48.

31. Dogan J, Jonasson J, Andersson E, Jemth P: Binding Rate Constants Reveal Distinct Features of Disordered Protein Domains. Biochemistry 2015, 54:4741-4750.

32. Levy ED, Kowarzyk J, Michnick SW: High-resolution mapping of protein concentration reveals principles of proteome architecture and adaptation. Cell Rep 2014, 7:1333-1340.

33. Klein P, Pawson T, Tyers M: Mathematical Modeling Suggests Cooperative Interactions between a Disordered Polyvalent Ligand and a Single Receptor Site. Curr Biol 2003, 13:1669-1678.

34. Locasale JW: Allovalency revisited: an analysis of multisite phosphorylation and substrate rebinding. $J$ Chem Phys 2008, 128:115106.

35. Iešmantavičius V, Dogan J, Jemth P, Teilum K, Kjaergaard M: Helical propensity in an intrinsically disordered protein accelerates ligand binding. Angew Chem Int Ed Engl 2014, 53:1548-1551.

36. Krieger JM, Fusco G, Lewitzky M, Simister PC, Marchant J, Camilloni C, Feller SM, De Simone A: Conformational recognition of an intrinsically disordered protein. Biophys $J$ 2014, 106:1771-1779.

37. Borcherds W, Theillet F-X, Katzer A, Finzel A, Mishall KM, Powell AT, Wu H, Manieri W, Dieterich $\mathrm{C}$, Selenko $\mathrm{P}$, et al.: Disorder and residual helicity alter p53-Mdm2 binding affinity and signaling in cells. Nat Chem Biol 2014, 10:1000-1002.

38. Crabtree MD, Borcherds W, Poosapati A, Shammas SL, Daughdrill GW, Clarke J: Conserved Helix-Flanking Prolines Modulate Intrinsically Disordered Protein:Target Affinity by Altering the Lifetime of the Bound Complex. Biochemistry 2017, 56:2379-2384.

39. Liu BA, Jablonowski K, Shah EE, Engelmann BW, Jones RB, Nash PD: SH2 domains recognize contextual peptide sequence information to determine selectivity. Mol Cell Proteomics MCP 2010, 9:2391-2404.

40. Stein A, Aloy P: Contextual specificity in peptide-mediated protein interactions. PloS One 2008, 3:e2524.

41. Frappier V, Duran M, Keating AE: PixelDB: Protein-peptide complexes annotated with structural conservation of the peptide binding mode. Protein Sci 2018, 27:276-285. 
42. Kelil A, Levy ED, Michnick SW: Evolution of domain-peptide interactions to coadapt specificity and affinity to functional diversity. Proc Natl Acad Sci U S A 2016, 113:E3862-3871.

43. Wang X, Bajaj R, Bollen M, Peti W, Page R: Expanding the PP2A Interactome by Defining a B56-Specific SLiM. Structure 2016, 24:2174-2181.

44. Palopoli N, González Foutel NS, Gibson TJ, Chemes LB: Short linear motif core and flanking regions modulate retinoblastoma protein binding affinity and specificity. Protein Eng Des Sel 2018, 31:69-77.

*45. Sundell GN, Arnold R, Ali M, Naksukpaiboon P, Orts J, Güntert P, Chi CN, Ivarsson Y: Proteome-wide analysis of phospho-regulated PDZ domain interactions. Mol Syst Biol 2018, 14:e8129.

The paper describes a novel method using peptide phage display for exploring phospho-regulated motif-based interactions on a large.scale. The approach simultaneously provides information on motif-based interactions and their potential site-specific regulation by Ser/Thr phosphorylation.

46. Zarrinpar A, Park S-H, Lim WA: Optimization of specificity in a cellular protein interaction network by negative selection. Nature 2003, 426:676-680.

47. Gibson TJ, Dinkel H, Van Roey K, Diella F: Experimental detection of short regulatory motifs in eukaryotic proteins: tips for good practice as well as for bad. Cell Commun Signal 2015, 13:42.

*48. Krystkowiak I, Davey NE: SLiMSearch: a framework for proteome-wide discovery and annotation of functional modules in intrinsically disordered regions. Nucleic Acids Res 2017, 45:W464-W469.

The authors devise a user-friendly discovery tool that search a proteome of interest for a given SLiM to discover putative novel cases of functionals. The web-based tool provides ample information about the the putative motifs, such as conservation, accessibility, and post-translational modification.

49. Erdős G, Szaniszló T, Pajkos M, Hajdu-Soltész B, Kiss B, Pál G, Nyitray L, Dosztányi Z: Novel linear motif filtering protocol reveals the role of the LC8 dynein light chain in the Hippo pathway. PLoS Comput Biol 2017, 13:e1005885.

50. Bessette PH, Rice JJ, Daugherty PS: Rapid isolation of high-affinity protein binding peptides using bacterial display. Protein Eng Des Sel 2004, 17:731739.

51. Volkmer R, Tapia V, Landgraf C: Synthetic peptide arrays for investigating protein interaction domains. FEBS Lett 2012, 586:2780-2786. 
52. Ivarsson Y, Arnold R, McLaughlin M, Nim S, Joshi R, Ray D, Liu B, Teyra J, Pawson $\mathrm{T}$, Moffat $\mathrm{J}$, et al.: Large-scale interaction profiling of PDZ domains through proteomic peptide-phage display using human and viral phage peptidomes. Proc Natl Acad Sci 2014, 111:2542-2547.

53. Blikstad C, Ivarsson Y: High-throughput methods for identification of protein-protein interactions involving short linear motifs. Cell Commun Signal 2015, 13:38.

54. Reich LL, Dutta S, Keating AE: SORTCERY-A High-Throughput Method to Affinity Rank Peptide Ligands. J Mol Biol 2015, 427:2135-2150.

55. Davey NE, Seo M-H, Yadav VK, Jeon J, Nim S, Krystkowiak I, Blikstad C, Dong D, Markova N, Kim PM, et al.: Discovery of short linear motif-mediated interactions through phage display of intrinsically disordered regions of the human proteome. FEBS J 2017, 284:485-498.

56. Lemonidis K, MacLeod R, Baillie GS, Chamberlain LH: Peptide array-based screening reveals a large number of proteins interacting with the ankyrinrepeat domain of the zDHHC17 S-acyltransferase. J Biol Chem 2017, 292:17190-17202.

*57. Kumar A, Manatschal C, Rai A, Grigoriev I, Degen MS, Jaussi R, Kretzschmar I, Prota AE, Volkmer R, Kammerer RA, et al.: Short Linear Sequence Motif LxxPTPh Targets Diverse Proteins to Growing Microtubule Ends. Structure 2017, 25:924-932.e4.

An exemplary study of the interaction between end-binding proteins and the LxxPTPh motif, including discovery through peptide SPOT array, structural characterization, biophysical affinity measurements, identification of additional non-specific interactions N-terminal of the motif, and prediction and validation of additional motifcontaining ligands.

58. Rogov VV, Stolz A, Ravichandran AC, Rios-Szwed DO, Suzuki H, Kniss A, Löhr F, Wakatsuki S, Dötsch V, Dikic I, et al.: Structural and functional analysis of the GABARAP interaction motif (GIM). EMBO Rep 2017, 18:1382-1396.

59. Barbar E, Nyarko A: Polybivalency and disordered proteins in ordering macromolecular assemblies. Semin Cell Dev Biol 2015, 37:20-25.

*60. Clark S, Myers JB, King A, Fiala R, Novacek J, Pearce G, Heierhorst J, Reichow SL, Barbar EJ: Multivalency regulates activity in an intrinsically disordered transcription factor. eLife 2018, 7.

The authors used a battery of biophysical, structural and molecular biology approaches to show how multivalency and low occupancy of LC8 binding "TQT" motifs within a disordered region regulate transcriptional activity. 
61. Fung HYJ, Birol M, Rhoades E: IDPs in macromolecular complexes: the roles of multivalent interactions in diverse assemblies. Curr Opin Struct Biol 2018, 49:36-43.

62. Kitov PI, Bundle DR: On the Nature of the Multivalency Effect: A Thermodynamic Model. J Am Chem Soc 2003, 125:16271-16284.

63. Zanier K, Charbonnier S, Sidi AOMO, McEwen AG, Ferrario MG, PoussinCourmontagne P, Cura V, Brimer N, Babah KO, Ansari T, et al.: Structural Basis for Hijacking of Cellular LxxLL Motifs by Papillomavirus E6 Oncoproteins. Science 2013, 339:694-698.

64. Hagai T, Azia A, Babu MM, Andino R: Use of host-like peptide motifs in viral proteins is a prevalent strategy in host-virus interactions. Cell Rep 2014, 7:1729-1739.

65. Kruse T, Biedenkopf N, Hertz EPT, Dietzel E, Stalmann G, López-Méndez B, Davey NE, Nilsson J, Becker S: The Ebola Virus Nucleoprotein Recruits the Host PP2A-B56 Phosphatase to Activate Transcriptional Support Activity of VP30. Mol Cell 2018, 69:136-145.e6.

66. Karlsson OA, Ramirez J, Öberg D, Malmqvist T, Engström Å, Friberg M, Chi CN, Widersten M, Travé G, Nilsson MTI, et al.: Design of a PDZbody, a bivalent binder of the E6 protein from human papillomavirus. Sci Rep 2015, 5:9382.

67. Ramirez J, Poirson J, Foltz C, Chebaro Y, Schrapp M, Meyer A, Bonetta A, Forster A, Jacob Y, Masson M, et al.: Targeting the Two Oncogenic Functional Sites of the HPV E6 Oncoprotein with a High-Affinity Bivalent Ligand. Angew Chem Int Ed Engl 2015, 54:7958-7962.

68. Mittag T, Orlicky S, Choy W-Y, Tang X, Lin H, Sicheri F, Kay LE, Tyers M, Forman-Kay JD: Dynamic equilibrium engagement of a polyvalent ligand with a single-site receptor. Proc Natl Acad Sci U S A 2008, 105:17772-17777.

69. Werner A, Baur R, Teerikorpi N, Kaya DU, Rape M: Multisite dependency of an $\mathrm{E} 3$ ligase controls monoubiquitylation-dependent cell fate decisions. eLife 2018, 7 .

70. Gordley RM, Bugaj LJ, Lim WA: Modular engineering of cellular signaling proteins and networks. Curr Opin Struct Biol 2016, 39:106-114.

71. Babu MM: The contribution of intrinsically disordered regions to protein function, cellular complexity, and human disease. Biochem Soc Trans 2016, 44:1185-1200.

72. Gee SH, Quenneville S, Lombardo CR, Chabot J: Single-amino acid substitutions alter the specificity and affinity of PDZ domains for their ligands. Biochemistry 2000, 39:14638-14646. 
73. Schneider S, Buchert M, Georgiev O, Catimel B, Halford M, Stacker SA, Baechi T, Moelling K, Hovens CM: Mutagenesis and selection of PDZ domains that bind new protein targets. Nat Biotechnol 1999, 17:170-175.

74. Panni S, Dente L, Cesareni G: In vitro evolution of recognition specificity mediated by SH3 domains reveals target recognition rules. J Biol Chem 2002, 277:21666-21674.

75. Wiedemann U, Boisguerin P, Leben R, Leitner D, Krause G, Moelling K, Volkmer-Engert R, Oschkinat H: Quantification of PDZ domain specificity, prediction of ligand affinity and rational design of super-binding peptides. $J$ Mol Biol 2004, 343:703-718.

76. Ernst A, Gfeller D, Kan Z, Seshagiri S, Kim PM, Bader GD, Sidhu SS: Coevolution of PDZ domain-ligand interactions analyzed by highthroughput phage display and deep sequencing. Mol Biosyst 2010, 6:1782.

77. Karlsson OA, Sundell GN, Andersson E, Ivarsson Y, Jemth P: Improved affinity at the cost of decreased specificity: a recurring theme in PDZ-peptide interactions. Sci Rep 2016, 6:34269.

78. Goldman A, Roy J, Bodenmiller B, Wanka S, Landry CR, Aebersold R, Cyert MS: The calcineurin signaling network evolves via conserved kinasephosphatase modules that transcend substrate identity. Mol Cell 2014, 55:422-435.

79. Cohen RD, Pielak GJ: A cell is more than the sum of its (dilute) parts: A brief history of quinary structure: A Brief History of Quinary Structure. Protein Sci 2017, 26:403-413.

80. Via A, Uyar B, Brun C, Zanzoni A: How pathogens use linear motifs to perturb host cell networks. Trends Biochem Sci 2015, 40:36-48.

*81. Zeng M, Ye F, Xu J, Zhang M: PDZ Ligand Binding-Induced Conformational Coupling of the PDZ-SH3-GK Tandems in PSD-95 Family MAGUKs. $J$ Mol Biol 2018, 430:69-86.

This paper and previous work from the laboratory use structural and biophysical methods to demonstrate how regions outside of the interaction region as well as neighboring domains and multimeric complexes modulate protein-protein interactions. 


\section{Figure legends:}

Figure 1: Schematic representations of different levels of how to achieve specificity in protein-protein interactions involving disordered binding motifs. (a) Side chain and backbone interactions between the core motif and the binding pocket of the folded recognition domain. (b) More or less specific interactions outside of the binding pocket can increase or decrease affinity for a certain binding motif (or vice versa). (c) Multivalency as exemplified by a bivalent interaction will increase affinity and specificity further both by increasing the frequency of productive collisions and by a second intramolecular interaction, which will increase the interaction surface and slow down dissociation since both contacts must break simultaneously. (d) Allovalency will occur since there is a certain probability of re-binding before the protein and ligand escape their common "sphere" and fully dissociates. This is particularly prevalent when multiple binding sites are involved, as exemplified here by three interaction motifs. (e, f) The place and time of expression of motifs and domains ensure that many non-specific interactions are avoided. 
